\title{
La cultura organizacional, un camino para humanizar la implementación del sistema de gestión de calidad - ISO 9001:2008*
}

\section{Organizational culture, a way to humanize the implementation of quality management systems- ISO 9001:2008}

Recibido: 15 de mayo de 2015

Jenny Astrid Barahona Pico ${ }^{* *}$

UNITRÓPICO

Deisy Rodríguez Araujo ***

Fundación Universitaria del Área Andina

\section{RESUMEN}

La pregunta que orientó la presente investigación inquirió por cómo lograr una cultura organizacional que propicie un ambiente adecuado para implementar un sistema de gestión de la calidad NTC ISO 9001:2008. Como complemento a lo anterior, se trazó el objetivo de elaborar una propuesta metodológica para que fuera una herramienta de fortalecimiento de la cultura organizacional en la implementación de NTC ISO 9001:2008 en dos empresas del sector de hidrocarburos. Se analizaron cuatro referentes conceptuales relevantes: formas de percepción, valores organizacionales, clima organizacional y la norma técnica NTC ISO 9001:2008. Para la recopilación de los datos se usó una encuesta. Los hallazgos más notables de la investigación son una invitación a comprender la necesidad de enfatizar en el entramado cultural de las organizaciones, cuando de la implementación de sistemas de gestión de la calidad se trata. En consecuencia, se propone como conclusión fundamental la necesidad de no instrumentalizar a los seres humanos en el curso de dichos procesos, a la vez que se sugiere su humanización.

\footnotetext{
Artículo de resultado de investigación.

** Licenciada en Química. Especialista en Análisis Químico Instrumental. Magíster en Calidad y Gestión Integral. Correo electrónico: yenny_pico@ hotmail.com

*** Psicóloga. Especialista en Gerencia del Talento Humano. Magíster en Calidad y Gestión Integral. Correo electrónico: dechy888@gmail.com
} 
Palabras clave: sistema de gestión de la calidad, clima laboral, cultura organizacional, valores organizacionales, ISO 9001.

\section{ABSTRACT}

The question which guided this research was how to achieve an organizational culture that fosters an adequate environment to implement an NTC ISO 9001: 2008 quality management system. As a complement to the above, the objective was to develop a methodological proposal to be a tool to strengthen the organizational culture in the implementation of NTC ISO 9001:2008 in two oil sector companies. Four relevant conceptual references were analyzed: perception forms, organizational values, organizational climate and technical standard NTC ISO 9001: 2008. The data were collected through a survey. The most notable findings of the research are an invitation to understand the need to emphasize in the cultural framework of organizations, when the implementation of quality management systems is treated, people not as a second order element but as central factor. Consequently, it is proposed as a fundamental conclusion the need to not instrumentalize human beings in the course of these processes, while suggesting their humanization

Keywords: Organizational culture, quality management system, work climate, organizational values, ISO 9001.

\section{INTRODUCCIÓN}

Uno de los problemas centrales a los que se enfrentan las compañías modernas cuando implementan un sistema integrado de calidad, está relacionado con los impactos que dicho procedimiento replica en la dinámica cultural de la empresa, al instrumentalizar a los empleados en lugar de considerar sus rasgos culturales.
Este trabajo sugiere entender la implementación de un sistema integrado de calidad como un proceso que interviene en la cultura organizacional, concibiendo el comportamiento de las personas, el activo más importante de la empresa, no como un simple ordenamiento técnico y operacional que se instrumentaliza en pasos de obligatorio cumplimiento, y por tanto se propone avanzar hacia la humanización de la implementación de cualquier sistema de gestión de la calidad (SGC).

Sin deslegitimar la validez del sistema de gestión de la calidad ISO 9001:2008, ni su aporte al desarrollo y competitividad de las organizaciones, se propone adoptar una cultura organizacional basada en las cualidades específicas de las personas.

Si bien el desarrollo de un SGC exige una serie de procedimientos de carácter técnico, aun así se presentan cuestiones de carácter emocional por parte de los empleados acerca del proceso del SGC, tales como ansiedad y miedo, además de un sinnúmero de situaciones que no se resuelven con la adopción de procedimientos estándar como en el caso del SGC, y que condicionan las relaciones humanas, dándole sentido a la cultura de las organizaciones, factores que pocas veces los expertos consideran.

Es importante referir el trabajo de Sánchez (2008), en el que se caracterizan y analizan los estilos de dirección y liderazgo de dos organizaciones. Para algunos la cultura organizacional es un potencial activo estratégico desde la perspectiva de la administración por su importancia para la implementación de la estrategia y sus efectos en la eficiencia y la efectividad del desempeño (Rodríguez, 2009). Para otros, como González y Fernández (2000), "la cultura de calidad de una organización se logra cuando sus miembros la han interiorizado" (p. 114). Por otra parte "los estilos de dirección para el siglo XXI demandan estar preparados para los cambios constantes, tanto para seguirlos como para 
generarlos" (Rosales, 1997, p. 154). En otras circunstancias autores como Omar y Urteaga (2010), analizaron las relaciones entre la cultura nacional y la cultura organizacional en 16 empresas argentinas, encontrando que las organizaciones nacionales comparadas con las privadas implementan prácticas orientadas a los empleados y a los sistemas rígidos; pero al compararlas de forma inversa, se implementan prácticas orientadas a los resultados, al mercado y a los sistemas abiertos.

Teniendo en cuenta lo anterior, esta investigación propone un mecanismo que modifique la percepción -del empleador- acerca del empleado frente a la implementación de SGC ISO 9001:2008 para potencializar su desempeño e igualmente su producción, aprovechando asílos beneficios de la cultura del buen trato.

Desde una perspectiva de carácter epistemológico, conocer la importancia que tiene la implementación de un SGC en el marco de la cultura organizacional, genera valor al proceso investigativo, en la medida en que es posible determinar los aspectos en los que se debe tener particular cuidado en lo atinente a preservar las relaciones humanas en los distintos ambientes de trabajo, permitiendo así que la organización avance hacia nuevas formas de proteger su recurso humano, lo que en el fondo se establece como conocimiento válido para la gestión efectiva de las organizaciones.

\section{METODOLOGÍA}

Durante esta investigación se utilizaron estrategias metodológicas basadas en un enfoque social, sustentadas en las perspectivas de la investigación social: el diseño, según Ibañez (2000), en las tres perspectivas: "distributiva, estructural y dialéctica", quien además cita que una dimensión referencial del componente simbólico permite expresar el lenguaje y la investigación de opiniones desde los protagonistas de los hechos, utilizando la encuesta como método de aplicación general. Este enfoque permitió realizar un análisis de las vicisitudes y sucesos expresados en las organizaciones objeto de estudio que proporcionó información, salvaguardando el principio de confidencialidad (Reyes, Ibáñez y Álvarez, 1992).

La dimensión vertical de la técnica apunta a los individuos supuestos pilares fijos del orden social, la dimensión horizontal apunta al juego del lenguaje (pregunta-respuesta) a que son sometidos (García, Ibáñez y Francisco, 2000 p. 70).

En el diseño de la encuesta aplicada se tomó como base lo expuesto por García, Ibáñez y Alvira (2000). Para construir dicho instrumento se realizó una revisión bibliográfica con información específica sobre la cultura organizacional para la implementación del SGC NTC ISO 9001:2008, que aportó a la investigación elementos de análisis que posteriormente se convirtieron en las categorías. El tipo de encuesta es descriptiva, la cual consiste en describir con precisión, normalmente mediante porcentajes y promedios, las características del fenómeno observado; como consecuencia de dicha descripción se pretende identificar y cuantificar con exactitud la relevancia de cada uno de los aspectos estudiados en la etapa exploratoria (Kuznik, Hurtado y Espinel, 2010).

La población objeto de la investigación estuvo compuesta por dos organizaciones pertenecientes al sector de construcción de obras mecánicas, eléctricas y civiles del ámbito hidrocarburos, donde la encuesta se dirigió exclusivamente a población administrativa de las organizaciones.

La muestra se obtuvo de manera aleatoria o probabilística, que garantiza la extrapolarización de los resultados, dado que brinda mayor certeza acerca de la representatividad de la población y sus características, considerando proporciones adecuadas (Morales, 2012). 


\section{RESULTADOS}

Teniendo en cuenta los resultados de la sistematización de la encuesta, se diseñaron tablas, cuyos valores respectivos corresponden a diversas subcategorías.
En la primera tabla se analizaron las formas de percepción de la calidad en las empresas encuestadas:

Tabla 1. Percepción de la calidad en la empresa.

\begin{tabular}{|l|c|c|c|c|c|c|c|c|c|c|c|c|c|c|c|}
\hline \multirow{2}{*}{ ÍTEMS } & \multicolumn{3}{|c|}{ A P } & \multicolumn{3}{c|}{ P } & \multicolumn{3}{c|}{ NP NN } & \multicolumn{3}{c|}{ N } & \multicolumn{3}{c|}{ T N } \\
\cline { 2 - 14 } & A & B & C & A & B & C & A & B & C & A & B & G & A & B & C \\
\hline $\begin{array}{l}\text { Los procesos de } \\
\text { calidad de la empresa } \\
\text { han transformado el } \\
\text { desempeño de su trabajo } \\
\text { de manera: }\end{array}$ & 7,0 & 13,5 & $\mathbf{3 0 , 2}$ & 45,6 & 67,5 & $\mathbf{5 6 , 5}$ & 40,3 & 18,9 & 29,6 & 5,2 & 0,0 & 2,6 & 1,7 & 0,0 & 0,8 \\
\hline $\begin{array}{l}\text { La diferencia entre lo que } \\
\text { espera de la empresa y } \\
\text { lo que recibe de ella en } \\
\text { cuanto a calidad es: }\end{array}$ & 7,0 & 13,5 & $\mathbf{1 0 , 2}$ & 52,6 & 62,1 & $\mathbf{5 7 , 3}$ & 21,0 & 21,6 & 21,6 & 19,3 & 2,7 & 11 & 0,0 & 0,0 & 0,0 \\
\hline $\begin{array}{l}\text { La implementación de } \\
\text { los SGC han cambiado } \\
\text { el servicio al cliente de } \\
\text { manera: }\end{array}$ & 12,2 & 18,9 & $\mathbf{1 5 , 6}$ & 50,8 & 72,9 & $\mathbf{6 1 , 9}$ & 28,0 & 8,1 & $\mathbf{1 8 , 0}$ & 5,2 & 0,0 & 2,6 & 3,5 & 0,0 & 1,7 \\
\hline
\end{tabular}

"Convenciones AP = Altamente Positivo P = Positivo NP NN = Ni Positivo Ni Negativo

$\mathrm{N}=$ Negativo $\quad \mathrm{TN}=$ Totalmente Negativo

$A=$ Empresa $A$

$\mathrm{B}=$ Empresa $\mathrm{B}$

$\mathrm{C}=$ Consolidado, es decir, el promedio entre $\mathrm{A}+\mathrm{B}$

Nota: la suma de las $5 \mathrm{C}$ de cada fila es un número que se aproxima a 100.

*Estas convenciones aplican para las demás tablas que contienen datos del sistema Likert.

Fuente: elaboración propia.

De la tabla 1 se interpreta que los extremos de la escala Likert no son significativos, y los resultados que pueden convertirse en signos de alarma o de tranquilidad oscilan en las categorías Negativo, Ni Positivo Ni Negativo y Positivo. Adicionalmente, en el caso de las distintas formas de percepción, puede decirse que es positiva, aunque estos porcentajes deberían ser mayores para que pasaran desapercibidos, puesto que si se realizara una suma de las otras tres categorías que no son positivas ni negativas, el porcentaje de percepción de los empleados de estas dos empresas es considerablemente alto.
Cabe señalar que los procesos de calidad de la empresa no han transformado el desempeño de los empleados, es decir, ha pasado desapercibido, toda vez que un 29 $\%$ opina que el cambio ni es positivo ni es negativo. Además, esta percepción es mayor en los empleados de la empresa $\mathrm{A}$.

Cambiando de aspecto, el clima organizacional es el tema correspondiente a la siguiente línea de análisis. Para ello, conviene mirar con detenimiento la tabla 2: 
Tabla 2. Formas de percepción de la aplicación del SGC.

\begin{tabular}{|c|c|c|c|c|c|c|c|c|c|c|c|c|c|c|c|}
\hline \multirow{2}{*}{ ÍTEMS } & \multicolumn{3}{|c|}{ AP } & \multicolumn{3}{|c|}{$\mathrm{P}$} & \multicolumn{3}{|c|}{ NP NN } & \multicolumn{3}{|c|}{$\mathbf{N}$} & \multicolumn{3}{|c|}{ TN } \\
\hline & $\mathrm{A}$ & B & $\mathrm{C}$ & $\mathrm{A}$ & B & $\mathrm{C}$ & $\mathrm{A}$ & B & $\mathrm{C}$ & $\mathrm{A}$ & $B$ & $\mathrm{C}$ & $\mathrm{A}$ & B & $\mathrm{C}$ \\
\hline $\begin{array}{l}\text { La implementación del SGC afecta la } \\
\text { interacción entre los miembros de la } \\
\text { organización de modo: }\end{array}$ & 3,5 & 5,4 & 4,4 & 49,1 & 64,8 & 56,9 & 43,8 & 16,2 & 30,0 & 3,5 & 10,8 & 7,1 & 0,0 & 2,7 & 1,3 \\
\hline $\begin{array}{l}\text { Los comportamientos y actitudes del } \\
\text { personal de la organización le aportan } \\
\text { al SGC de una forma: }\end{array}$ & 7,0 & 8,1 & 7,5 & 40,3 & 72,9 & 56,6 & 43,8 & 13,5 & 28,6 & 8,7 & 5,4 & 7,0 & 0,0 & 0,0 & 0,0 \\
\hline $\begin{array}{l}\text { Las relaciones entre los miembros de } \\
\text { la organización influye en los procesos } \\
\text { de calidad de manera: }\end{array}$ & 5,2 & 10,8 & 8,0 & 50,8 & 67,5 & 59,2 & 31,5 & 21,6 & 26 & 10,5 & 0,0 & 5,2 & 1,7 & 0,0 & 0,8 \\
\hline $\begin{array}{l}\text { La implementación de SGC ha } \\
\text { generado al interior de la organización } \\
\text { formas de comunicación: }\end{array}$ & 7,0 & 5,4 & 6,2 & 40,3 & 64,8 & 52,6 & 45,6 & 16,2 & 30,9 & 7,0 & 10,8 & 8,9 & 0,0 & 2,7 & 1,3 \\
\hline
\end{tabular}

Fuente: elaboración propia.

Según los resultados, los encuestados se inclinan hacia la opción negativa. Los valores más altos indican que la implementación del SGC NTC ISO 9001:2008 no es positiva ni negativa, y para muchos incluso es negativa. Adicionalmente, vale la pena resaltar que en una respuesta están de acuerdo los empleados de las dos empresas: "Los comportamientos y actitudes del personal de la organización no le aportan al sistema de gestión de la calidad de una forma totalmente negativa”. También se puede apreciar que mientras los valores en $\mathrm{B}$ son más elevados, en la categoría $\mathrm{P}$ los valores en A se elevan en la categoría NP NN.

Se puede apreciar que la implementación del SGC NTC ISO 9001:2008 genera actitudes ycomunicaciones negativas en algunos casos, en porciones superiores al $10 \%$, tratándose de empresas con más de doscientos empleados, este número es elevado. En ese sentido y por la homogeneidad de los resultados en las distintas categorías, puede decirse que tanto la implementación del SGC NTC ISO 9001:2008 como el aporte de quienes lo implementan, está condicionado, es decir, se complementan entre sí.

Conviene subrayar también que más de un $67 \%$ está de acuerdo en que si no se llevan bien entre sí, la implementación del SGC no estaría cumpliendo con su propósito. De la siguiente categoría se deriva la tabla 3:

Tabla 3. Clima organizacional en la aplicación del SGC.

\begin{tabular}{|c|c|c|c|c|c|c|c|c|c|c|c|c|c|c|c|}
\hline \multirow{2}{*}{ ÍTEMS } & \multicolumn{3}{|c|}{ AP } & \multicolumn{3}{|c|}{$\mathrm{P}$} & \multicolumn{3}{|c|}{ NP NN } & \multicolumn{3}{|c|}{$\mathrm{N}$} & \multicolumn{3}{|c|}{$\mathrm{TN}$} \\
\hline & A & B & $\mathrm{C}$ & A & B & $\mathrm{C}$ & $\mathrm{A}$ & B & $\mathrm{C}$ & A & B & $\mathrm{C}$ & A & B & $\mathrm{C}$ \\
\hline $\begin{array}{l}\text { El entorno laboral que propicia } \\
\text { su organización para el } \\
\text { desarrollo de su trabajo es: }\end{array}$ & 14,0 & 16,2 & 15,1 & 47,3 & 72,9 & 60,1 & 17,5 & 10,8 & 14,1 & 19,3 & 0,0 & 9,6 & 1,7 & 0,0 & 0,8 \\
\hline
\end{tabular}




\begin{tabular}{|l|c|c|c|c|c|c|c|c|c|c|c|c|c|c|c|}
\hline \multirow{2}{*}{\multicolumn{1}{|c|}{ ÍTEMS }} & \multicolumn{3}{|c|}{ A P } & \multicolumn{4}{c|}{ P } & \multicolumn{3}{c|}{ NP NN } & \multicolumn{4}{c|}{ N } & \multicolumn{3}{c|}{ T N } \\
\cline { 2 - 13 } & A & B & G & A & B & C & A & B & C & A & B & C & A & B & C \\
\hline $\begin{array}{l}\text { En las decisiones que tienen } \\
\text { que ver con construcción de } \\
\text { espacios organizacionales } \\
\text { agradables su opinión es tenida } \\
\text { en cuenta de manera: }\end{array}$ & 1,7 & 8,1 & 4,9 & 35,0 & 56,7 & 45,9 & 45,6 & 35,1 & 40,3 & 15,7 & 0,0 & 7,8 & 1,7 & 0,0 & 0,8 \\
\hline
\end{tabular}

Fuente: elaboración propia.

A nivel general en las dos empresas el entorno laboral es positivo, y en parte se debe a que al construirse nuevos espacios se cuenta con la opinión de los empleados para que sean agradables. No obstante, se considera en este estudio que el porcentaje $(9,85)$ que califica estos espacios como negativos y que la opinión no es tenida en cuenta para que sean agradables $(7,89)$, genera preocupaciones que deberín analizarse mejor por parte de los organizadores de los espacios.
En la tabla 4 está el resultado de los valores organizacionales al estilo de dirección, se lee que los empleados de la empresa A tienen percepciones negativas acerca de estos estilos de dirección. Es de resaltar la indiferencia que caracteriza a los encuestados acerca de las decisiones sobre el desempeño profesional que toma la alta dirección. La opinión no incide.

Tabla 4. Estilos de dirección.

\begin{tabular}{|l|r|c|c|c|c|c|c|c|c|c|c|c|c|c|c|}
\hline \multirow{2}{*}{ ÍTEMS } & \multicolumn{3}{|c|}{ A P } & \multicolumn{4}{|c|}{ P } & \multicolumn{3}{c|}{ NP NN } & \multicolumn{3}{c|}{ N } & \multicolumn{3}{c|}{ T N } \\
\cline { 2 - 15 } & A & B & C & A & B & C & A & B & C & A & B & C & A & B & C \\
\hline $\begin{array}{l}\text { Los líderes de su organización } \\
\text { utilizan un estilo de dirección: }\end{array}$ & 5,2 & 18,9 & $\mathbf{1 2 , 0}$ & 38,6 & 64,8 & $\mathbf{5 1 , 7}$ & 35,0 & 16,2 & 25,6 & 15,7 & 0,0 & 7,8 & 5,2 & 0,0 & 2,6 \\
\hline $\begin{array}{l}\text { En las decisiones que toma la alta } \\
\text { dirección que tienen que ver con su } \\
\text { desempeño profesional, su opinión } \\
\text { es valorada de una manera: }\end{array}$ & 1,7 & 13,5 & 7,6 & 36,8 & 59,4 & 48,1 & 49,1 & 24,3 & 36,7 & 10,5 & 2,7 & 6,6 & 1,7 & 0,0 & 0,8 \\
\hline
\end{tabular}

Fuente: elaboración propia.

Del mismo modo los planes de mejoramiento que proponen ejecutivos de A y B son la mayoría de veces indiferentes a la opinión de los empleados.

Continuando con el análisis de resultados, hay una subcategoría denominada requisitos generales que se puede apreciar en la tabla 5. Según esta, para más del $33 \%$ de la muestra no existe un significado positivo atribuido al SGC NTC ISO 9001:2008 por los miembros de la organización. A esto se suma que más del
$40 \%$ no considera positiva la información que recibe respecto al SGC NTC ISO 9001:2008. Además, los cambios realizados a partir de la implementación del SGC no generan importancia en más del $25 \%$ de ellos. Para finalizar este análisis se encuentra en la tabla 5 que el seguimiento realizado al SGC NTC ISO 9001:2008 ni es positivo, ni negativo, pero si se le suma el 10,6 que lo considera negativo, este factor es preponderante. 
Tabla 5. Requisitos generales.

\begin{tabular}{|c|c|c|c|c|c|c|c|c|c|c|c|c|c|c|c|}
\hline \multirow{2}{*}{ ÍTEMS } & \multicolumn{3}{|c|}{ AP } & \multicolumn{3}{|c|}{$\mathrm{P}$} & \multicolumn{3}{|c|}{ NP NN } & \multicolumn{3}{|c|}{$\mathrm{N}$} & \multicolumn{3}{|c|}{ TN } \\
\hline & A & B & $\mathrm{C}$ & A & B & $\mathrm{C}$ & A & B & C & A & B & $\mathrm{C}$ & A & B & C \\
\hline $\begin{array}{l}\text { El significado que los miembros de la } \\
\text { organización le atribuyen al SGC es: }\end{array}$ & 7,0 & 13,5 & 10,2 & 45,6 & 64,8 & 55,2 & 36,8 & 18,9 & 27,8 & 10,5 & 2,7 & 6,6 & 0,0 & 0,0 & 0,0 \\
\hline $\begin{array}{l}\text { Cómo calificaría la información que } \\
\text { usted recibe respecto al SGC NTC } \\
\text { ISO 9001: }\end{array}$ & 3,5 & 5,4 & 4,4 & 35,0 & 70,2 & 52,6 & 38,6 & 21,6 & 30,1 & 17,5 & 2,7 & 10,1 & 5,2 & 0,0 & 2,6 \\
\hline $\begin{array}{l}\text { ¿Considera que el seguimiento que se } \\
\text { hace al SGC NTC ISO } 9001 \text { es?: }\end{array}$ & 8,7 & 8,1 & 8,4 & 36,8 & 70,2 & 53,5 & 33,3 & 16,2 & 24,7 & 15,7 & 5,4 & 10,6 & 5,2 & 0,0 & 2,6 \\
\hline
\end{tabular}

Fuente: elaboración propia.

Finaliza la escala Likert con la subcategoría denominada recurso humano.

Tabla 6. Recursos humanos.

\begin{tabular}{|c|c|c|c|c|c|c|c|c|c|c|c|c|c|c|c|}
\hline \multirow{2}{*}{ ÍTEMS } & \multicolumn{3}{|c|}{ AP } & \multicolumn{3}{|c|}{$P$} & \multicolumn{3}{|c|}{ NP NN } & \multicolumn{3}{|c|}{$\mathrm{N}$} & \multicolumn{3}{|c|}{ TN } \\
\hline & $\mathrm{A}$ & B & C & $\mathrm{A}$ & B & $\mathrm{C}$ & $\mathrm{A}$ & $\mathrm{B}$ & $\mathrm{C}$ & A & B & $\mathrm{C}$ & $\mathrm{A}$ & B & $\mathrm{C}$ \\
\hline $\begin{array}{l}\text { La infraestructura con la que } \\
\text { cuenta para el desempeño de sus } \\
\text { funciones es: }\end{array}$ & 24,5 & 16,2 & 20,3 & 54,3 & 70,2 & 62,3 & 14,0 & 8,1 & 11,0 & 7,0 & 5,4 & 6,2 & 0,0 & 0,0 & 0,0 \\
\hline $\begin{array}{l}\text { Las capacitaciones planeadas } \\
\text { por su empresa para fortalecer su } \\
\text { capacidad de desempeño son: }\end{array}$ & 10,5 & 23,3 & 16,9 & 52,6 & 66,6 & 59,6 & 26,3 & 10,0 & 18,1 & 0,0 & 0,0 & 0,0 & 10,5 & 0,0 & 5,2 \\
\hline $\begin{array}{l}\text { El ambiente de trabajo de la } \\
\text { empresa en la que usted labora es: }\end{array}$ & 8,9 & 24,3 & 16,6 & 55,3 & 64,8 & 60,1 & 16,0 & 8,1 & 12,0 & 19,6 & 2,7 & 11,1 & 0,0 & 0,0 & 0,0 \\
\hline
\end{tabular}

Fuente: elaboración propia.

De la tabla 6 llama la atención que el 6,21 \% de los encuestados considera negativa la infraestructura con la que cuenta para el desempeño de sus funciones. En la empresa $A$ en un $10,53 \%$ se estima como totalmente negativo el proceso de capacitación que recibe por parte de la empresa. De igual forma, es negativo el ambiente de trabajo que se vive allí para el 11,17\% de los encuestados.

A continuación se hace el análisis de las preguntas que no tienen una estructura linkert. En la tabla 7 se relacionan los resultados obtenidos de las dos empresas encuestadas. De la pregunta por los valores que aporta el SGC NTC ISO 9001:2008 a la organización, se puede inferir que las opciones a, b y c son bien relevantes para las dos empresas, además, cobra especial valor la calidad en los procesos.

En cuanto a la pregunta por la vivencia de los valores dentro de la organización, se deduce que para las dos empresas la aplicación de valores se da de manera espontánea, mientras que un 21,05\% de los empleados 
de la empresa A consideran que no existen ese tipo de valores en la organización a la que pertenecen. Los empleados de las empresas encuestadas coinciden en que el valor que más deben practicar para el fortalecimiento del SGC NTC ISO 9001:2008 es el compromiso con el desempeño de sus funciones, seguido en menor intensidad por el de cumplimiento del contenido de la política de calidad de la empresa. Por otra parte, es llamativo que las organizaciones no adelanten acciones para promocionar los valores institucionales, así lo deja ver el $25,51 \%$ de los empleados, siendo más marcada esta concepción en los empleados de la empresa A.

Tabla 7. Información complementaria para configurar la implementación de un SGC.

\begin{tabular}{|c|c|c|c|c|}
\hline \multirow{2}{*}{ PREGUNTA } & \multirow{2}{*}{ OPCIONES DE RESPUESTA } & \multicolumn{2}{|c|}{ \% EMPRESAS } & \multirow{2}{*}{ PROM } \\
\hline & & $\mathrm{A}$ & B & \\
\hline \multirow{5}{*}{$\begin{array}{l}\text { ¿Cuáles considera usted son los } \\
\text { valores que le aporta el SGC a la } \\
\text { organización?: }\end{array}$} & a. Calidad en los procesos & 53,57 & 29.73 & 41.65 \\
\hline & b. Comunicación efectiva & 0,00 & 0,00 & 0,00 \\
\hline & c. Respeto por el cliente interno y externo & 1,79 & 2,70 & 2,24 \\
\hline & d. Las tres anteriores (a, b y c) & 44,64 & 64,86 & 54,75 \\
\hline & e. Otros. ¿Cuáles? & 0,00 & 2,70 & 1,35 \\
\hline \multirow{5}{*}{$\begin{array}{l}\text { La construcción de los valores } \\
\text { como el respeto, tolerancia, } \\
\text { responsabilidad, honestidad, } \\
\text { entre otros, en su organización } \\
\text { se viven de una manera: }\end{array}$} & a. Impuesta & 5,26 & 2,70 & 3,98 \\
\hline & b. Planeada & 24,56 & 32,43 & 28,49 \\
\hline & c. Espontáneamente & 49,12 & 62,16 & 55,64 \\
\hline & d. No hay valores institucionales & 21,05 & 0,00 & 10,52 \\
\hline & e. Otros. ¿Cuáles? & 0,00 & 2,70 & 1,35 \\
\hline \multirow{5}{*}{$\begin{array}{l}\text { ¿Cuál considera debe ser el } \\
\text { valor más importante que deben } \\
\text { practicar los empleados para el } \\
\text { fortalecimiento del SGC?: }\end{array}$} & a. Cumplir la política de calidad de la organización & 26,32 & 24,32 & 25,32 \\
\hline & $\begin{array}{l}\text { b. Ser responsable en los procedimientos que indican los } \\
\text { manuales de funciones }\end{array}$ & 8,77 & 18,92 & 13,84 \\
\hline & c. Comprometerse con el desempeño de sus funciones & 40,35 & 51,35 & 45,85 \\
\hline & d. Respeto con los clientes externos e internos (trabajadores) & 21,05 & 2,70 & 11,87 \\
\hline & e. Otros. ¿Cuáles? & 3,51 & 2,70 & 3,10 \\
\hline \multirow{2}{*}{$\begin{array}{l}\text { ¿Se entera usted de las } \\
\text { decisiones que se toman en la } \\
\text { organización?: }\end{array}$} & Cuenta de SÍ & 56 & 78 & 67 \\
\hline & Cuenta de NO & 44 & 22 & 33 \\
\hline \multirow{5}{*}{$\begin{array}{l}\text { Si su respuesta es afirmativa, ¿aa } \\
\text { través de qué medio se entera } \\
\text { de las decisiones?: }\end{array}$} & a. Correos electrónicos masivos & 65,63 & 75,86 & 70,74 \\
\hline & b. Comunicados en papel & 0,00 & 3,45 & 1,72 \\
\hline & c. Comunicación humana - directa & 28,13 & 17,24 & 22,68 \\
\hline & d. No recibe información en este sentido & 6,25 & 3,45 & 4,85 \\
\hline & e. Otros medios. ¿Cuáles? & 0,00 & 0,00 & 0,00 \\
\hline
\end{tabular}

Fuente: elaboración propia.

Finalmente es alto el porcentaje de empleados que no se entera de las decisiones que toman sus empresas y quienes lo hacen, el medio más frecuente para hacerlo es el correo electrónico. 


\section{DISCUSIÓN}

De acuerdo con lo expuesto por Goodenough (1957), la percepción depende de la sensación. El perceptor tiene consciencia de objetos y eventos en su ambiente y no meramente impresiones sensoriales. Esto los convierte en interpretadores de su realidad con plena consciencia de lo que sienten, perciben y experimentan.

Deming y Medina (1989), demostraron la relación existente entre productividad y calidad, estableciendo que "el incremento de la calidad reduce los costes por una reducción de los fallos, por una reducción de los reprocesos, por una reducción de los desechos". Teniendo en cuenta lo anterior, se especifica que el desempeño está asociado a la productividad y a la transformación de lo que se hace a partir de un nuevo proceso, lo cual provoca cambios positivos y negativos, considerando la persona que los asimila.

Por otro lado, la idea de calidad puede analizarse mediante el esfuerzo de los responsables del SGC, puesto que este constituye "la relación entre el potencial de servicio y la prestación real" (Etkin, 2006, p. 415). En cuanto a cultura, Goodenough (1957) no la concibe como algo que se pueda cuantificar dado que es inerte y carente de cuerpo.

En otro sentido, la categoría clima organizacional contempla dos subcategorías: comunicación y estilos de dirección; en ese orden de ideas, dice Munduate (1997) que "cuando en las organizaciones hay una buena cultura organizacional, esta se concibe como realidades construidas socialmente, en donde los individuos son moldeados por el contexto cultural más amplio". Misas (2004) precisa:

Cuando las organizaciones sufren ataques de su entorno, tienden a crear sólidos mecanismos de protección que las blindan contra las agresiones externas, mediante la aparición de culturas internas defensivas que al mismo tiempo las tornan insensibles a los cambios del entorno (p. 169).
En el texto de Romo (2008), se cita a Nosnik para advertir que la comunicación es lineal, además: causal, privilegiada y restringida y donde la fuente tiene mayor acceso a información privilegiada. De la misma manera Rosales (1997) argumenta cómo el modelo de administración en una organización, o un estilo de dirección determinado puede ser predominante dentro de los comportamientos y actitudes de los otros líderes.

Por su parte, González y Fernández (2000) mencionan que la cultura organizacional requiere un trabajo gerencial de mayor importancia. Los directivos deben preservar las glorias del pasado que agregan valor y encauzar la dirección del cambio para eliminar los componentes culturales indeseables. Desde esta perspectiva se deduce que el estilo de dirección de una organización está directamente ligado con el clima laboral, dado que las personas conforman los ambientes que habitan y sus formas de actuar generan emociones dentro de los espacios. Al respecto, Toro (2001) establece que el clima organizacional es un determinante clave para fenómenos significativos de la vida organizacional, como son la productividad y la competitividad. Sin embargo, la cultura organizacional, según plantea García (2001), "es un sistema de valores compartidos y de creencias en interacción con la población de una empresa, sus estructuras organizacionales y sus sistemas de control para producir normas de comportamiento".

Finalmente, Gordon (1991) (citado por Paramo, 2001) señala que la cultura organizacional se halla fuertemente influenciada por las características de la industria en la cual la organización empresarial realiza su operación, que para este caso es el sector construcción. De esa manera se discurre sobre el papel de los empleados, el cual oscila entre cumplir la política de calidad de la organización, ser responsables en los procedimientos que indican los manuales de funciones, comprometerse con el desempeño de sus funciones y el respeto con los clientes internos y externos, todo para el fortalecimiento del Sistema Integrado de Calidad. 
La propuesta de la investigación surgió como resultado de las encuestas aplicadas en las dos organizaciones y tiene dos enfoques: uno didáctico, el cual consiste en una propuesta de aprendizaje experiencial, donde se establece el aprendizaje por descubrimiento y está relacionado con que las personas aprendan en el saber hacer; y otro pedagógico, el cual tiene como propósito que las personas cambien su pensamiento o manera de actuar frente a una situación específica. En síntesis, cuando la gente aprende asume prácticas culturales que le sirven para darle nuevos significados a los sentidos de las cosas y que se instalan en su saber cotidiano.

\section{CONCLUSIONES}

Se encuentra que en las organizaciones investigadas no hay frecuencia en las capacitaciones planteadas, que tal vez responden a planes inmediatistas y no a un verdadero diagnóstico de necesidades que permita darle a la gente lo que necesita. Las decisiones respecto al desempeño profesional de los trabajadores no surgen de consultas, partiendo de imposiciones y no de una construcción conjunta.

Es evidente que las dos organizaciones cuentan con climas organizacionales totalmente distintos, pero coincidieron en que no existen espacios organizacionales que los involucren en la construcción de estos. Los valores organizacionales no son reconocidos; de la misma manera, los valores del SGC NTG ISO 9001:2008 no se identifican, alejando a las personas de una verdadera consciencia del aporte de una nueva forma de hacer.

Es importante concienciar a las personas implicadas en un proceso de certificación sobre los cambios que se anteponen cuando se implementa un nuevo proceso. Los métodos de cambio resultan traumáticos para las personas porque implican nuevas formas de hacer, pero la dinámica del proceso puede cambiar en la medida en que se busquen mecanismos como la sensibilización paulatina.

Los procesos de calidad deben transformar el desempeño de las personas de una forma positiva. Esto se logra cuando se realiza una sensibilización del proceso de manera progresiva y no se impone 0 se vende la implementación como una actividad alterna que se incluye dentro de las establecidas.

Las empresas objeto de estudio deben aunar todos sus esfuerzos particulares en la construcción de estrategias de medición y seguimiento de la percepción del cliente interno y externo frente a la calidad. Estas no se deben convertir en estrategias inmediatistas o para el cumplimiento de una auditoría. Deben trascender y encontrar los correctivos necesarios para que se le dé movimiento a un círculo de retroalimentación, plan de mejora y cambios. Además, se hace necesario avanzar en el estudio de metodologías aplicadas que permitan orientar a las personas en la implementación de un SGC, dirigidas a los valores, al manejo del clima, que profundicen las formas de percibir su realidad y le aporten al cambio.

\section{REFERENCIAS}

Deming, E., y Medina, J. N. (1989). Calidad, productividad y competitividad: la salida de la crisis. Día de Santos, 1(1), 94-128.

Etkin, J. (2006). Gestión de la complejidad en las organizaciones. La estrategia frente a los imprevisto y lo impensado. Buenos Aires: Ediciones Granica S. A.

García de la Torre, C. (2001). Una aproximación a los estudios interculturales en la Admininstración. Administración y Organizaciones, 6(3), 49-82. 
Garcí, F., Ibáñez, J., y Francisco, A. (2000). El análisis de la realidad social: métodos y técnicas de investigación. Alianza, 64.

González, A., y Fernández, E. M. (2000). La cultura de la organización en la gestión total de la calidad. Ensaios e Ciencia: Ciencias Biologicas, Agrarias e da Saúde, 4(3), 99-114.

Goodenough, W. H. (1957). The componential analysis of kinship. Language, 132-167.

Ibáñez, J. (2000). Perspectivas de la investigación social: el diseño en las tres perspectivas. Madrid: Alianza.

Kuznik, A., Hurtado, A., y Espinel, A. (2010). El uso de la encuesta de tipo social en traductología. Características metodológicas. Redalyc, (2), 6.

Misas, G. (2004). La educación superior en Colombia. Análisis y estrategias para su desarrollo. Bogotá, Colombia: Universidad Nacional de Colombia.

Morales, P. (2012, 13 de diciembre). Estadística aplicada a las Ciencias Sociales. Tamaño necesario de la muestra: ¿Cuántos sujetos necesitamos? Recuperado de http://www2.df.gob.mx/virtual/evaluadf/docs/ gral/taller2015/S0202EAC.pdf

Munduate, L. (1997). Psicología social de la organización. Las personas organizando. Madrid: Pirámide.
Omar, A., y Florencia, A. (2010, enero-abril). El impacto de la cultura nacional sobre la cultura organizacional. Universitas Psychologica, 9(1), 79-92.

Paramo, D. (2001, junio). Hacia la construcción de un modelo de cultura organizacional orientada al mercado. Revista Colombiana de Marketing, 2(6), $1-26$.

Reyes, R., Ibáñez, J., y Álvarez, F. (1992). Las ciencias sociales en España. Historia inmediata, críticas y perspectivas. Madrid: Universidad Complutense.

Rodríguez, R. (2009). La cultura organizacional, un potencial estratégico desde la perspectiva de la administración. Invenio, 12(22), 67-92.

Rosales, R. (1997). Estilos de dirección y clima organizacional. Ciencias Sociales, 141-154.

Sánchez, I. D. (2008). Los estilos de dirección y liderazgo, propuesta de un modelo de caracterización y análisis. Pensamiento y Gestión, (25), 1-39.

Soria, R. (2008). Comunicación organizacional: un modelo aplicable a la microempresa. TEACS, 9-17.

Toro, F. (2001). El clima organizacional. Perfil de empresas colombianas. Medellín, Colombia: Cincel. 